\title{
A Waveguide-Coupled Colloidal Quantum Dot LED on a Silicon Nitride Platform
}

\author{
Lukas Elsinger $^{\dagger, \#}$, Ivo Tanghe ${ }^{\dagger, \#}$, Frederik Van Acker ${ }^{\star, \#}$, Natalia K. Zawacka ${ }^{\S, \#, ~}$

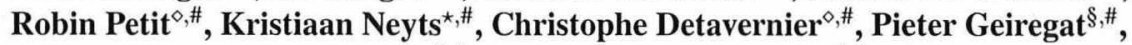 \\ Zeger Hens ${ }^{\S, \#}$ and Dries Van Thourhout ${ }^{\dagger, \#}$ \\ ${ }^{\dagger}$ Photonics Research Group, Ghent University - imec, 9052 Ghent, Belgium \\ ${ }^{\#}$ NB Photonics, Ghent University, 9052 Ghent, Belgium \\ ${ }^{\circ}$ Conformal Coating of Nanomaterials Group, Ghent University, 9000 Ghent, Belgium \\ ${ }^{\star}$ Liquid Crystal and Photonics Group, Ghent University, 9052 Ghent, Belgium \\ $\S$ Physics and Chemistry of Nanostructures Group, Ghent University, 9000 Ghent, Belgium \\ lukas.elsinger@ugent.be
}

\begin{abstract}
Colloidal quantum dots (QDs) have become an attractive light source for visible photonics. Here, we demonstrate the first integrated LED based on CdSe/CdS QDs, with the emission directly coupled to a silicon nitride waveguide. (C) 2020 The Author(s) OCIS codes: $130.0130,130.0250,130.3120,130.6622$
\end{abstract}

\section{Introduction}

Colloidal quantum dots (QDs) offer several advantages compared to traditional III-V materials for visible photonics, in particular their widely tunable emission wavelength, inexpensive wet-chemical synthesis and straightforward hybrid integration can make the difference. With hybrid LED designs, using organic materials for hole injection and $\mathrm{ZnO}$ nanocrystals for electron injection, internal quantum efficiencies approaching $100 \%$ have been demonstrated [1]. However, until now it has been challenging to find an appropriate waveguide layer, facilitating their integration on chip-based platforms. In this work we present the, to the best of our knowledge, first waveguide-coupled LED based on colloidal QDs.

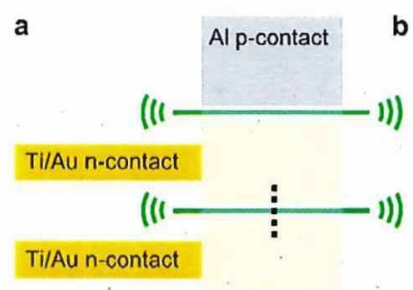

\section{d}
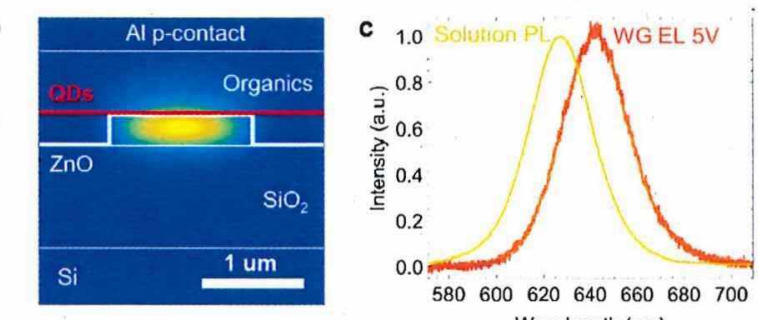

Wavelength $(\mathrm{nm})$

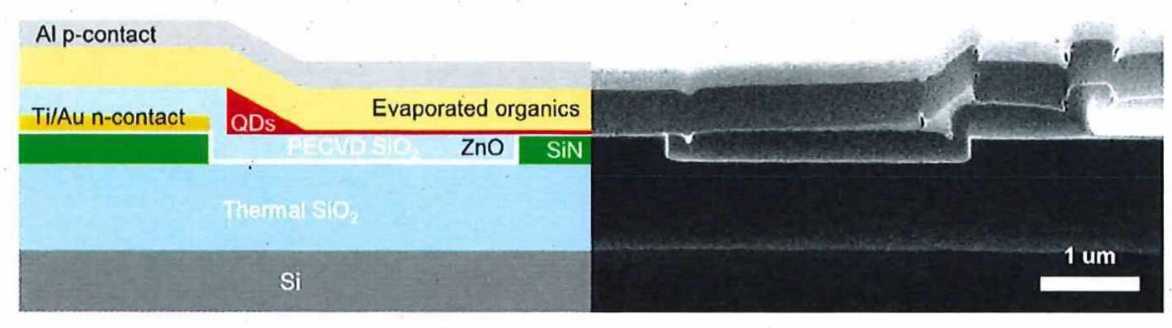

Fig. 1. (a) Schematic top-view of the device, the dashed line is indicating the position of the crosssection. (b) Simulated mode profile of the fundamental TE mode. (c) Photoluminescence (PL) spectrum of the CdSe/CdS QDs in solution and the device emission spectrum. (d) SEM image of a focused ion-beam cross-section next to a schematic drawing of the device.

\section{Design}

Unlike most high efficiency so-called inverted LED designs [1], we do not use a combination of a transparent conductive oxide and $\mathrm{ZnO}$ nanocrystals for electron injection. Instead, we employ a $15 \mathrm{~nm}$ thin layer of polycrys- 


\section{CONFIDENTIAL}

talline $\mathrm{ZnO}$ deposited by atomic layer deposition (ALD), which is contacted with a Ti/Au n-contact electrode next to the trench defining the silicon nitride waveguide (see Fig. 1). The ALD ZnO serves a dual purpose of electron transport and injection and was optimized for minimal optical loss $(-7 \mathrm{~dB} / \mathrm{cm})$. For the evaporated organics we used a combination of TCTA, NPB, and HAT-CN for band-alignment, hole transport and injection. The total layer thickness was increased to reduce the optical loss due the aluminum p-contact to an acceptable value $(-3 \mathrm{~dB} / \mathrm{cm})$.

\section{Measurements}

Figure 1(c) shows the output spectrum of a device at $5 \mathrm{~V}$ bias, for this measurement the sample was cleaved and the emission collected with a multimode fiber, in order to eliminate any influence of the grating coupler transmission spectrum. There is a clear red-shift of the spectrum, compared to the photoluminescence (PL) emission of the same $\mathrm{CdSe} / \mathrm{CdS}$ QD sample in solution. When increasing the bias voltage, we did not observe a significant shift of the electroluminescence (EL) spectrum. Both electrical and optical turn-on voltage were found to be approximately $2.5 \mathrm{~V}$ (see Fig. 2) and using a reverse bias, the device also worked as a detector. The maximum current density of $0.15 \mathrm{~A} \mathrm{~cm}^{-2}$ observed for our devices is low compared to values of up to $20 \mathrm{~A} \mathrm{~cm}^{-2}$ reported in literature [2]. We believe this is due to pinholes present in the aluminum p-contact, which lead to a degradation of the organic layers when performing measurements outside of an inert atmosphere. Therefore, we expect a significantly improved device performance after resolving this issue by improving the processing.
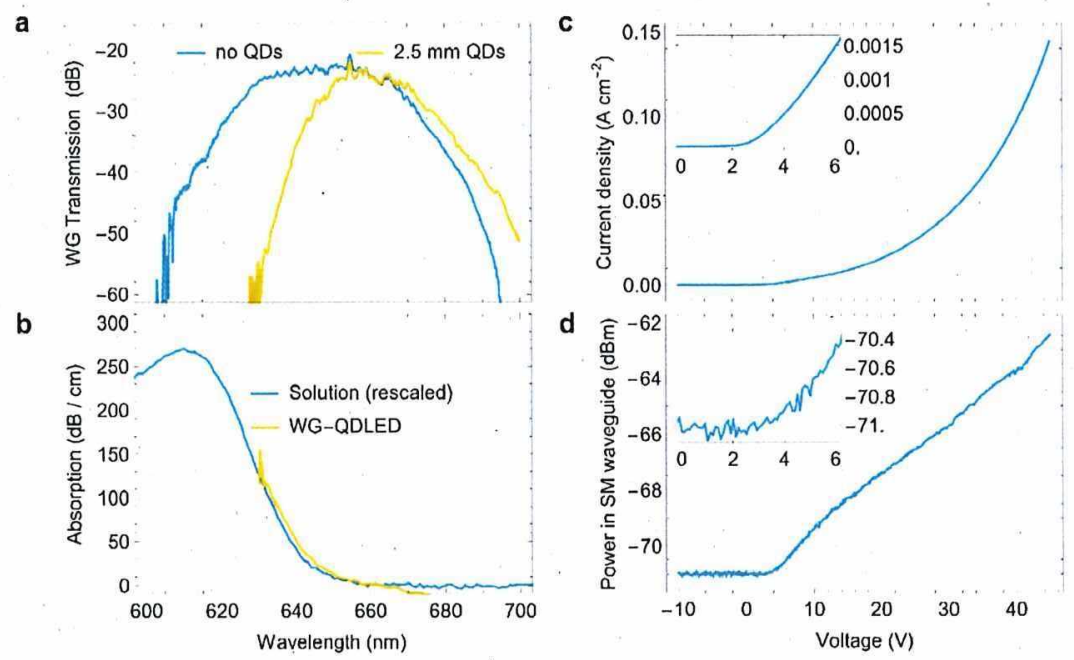

Fig. 2. (a) Waveguide transmission with and without QDs measured using two grating couplers. (b) Absorption spectrum of the QD layer extracted from the transmission measurement and from a measurement in solution. (c) Electrical and (d) electro-optic characteristics of the device.

\section{Outlook}

We anticipate that the demonstrated waveguide-coupled QD LEDs can find an application in chip-based absorption spectroscopy, as they can be post-processed on virtually any chip-based waveguide platform, at a low cost. In addition, we believe that our approach provides the missing low-loss waveguide layer, necessary for building an electrically pumped laser using colloidal QDs.

\section{References}

1. B. S. Mashford, M. Stevenson, Z. Popovic, C. Hamilton, Z. Zhou, C. Breen, J. Steckel, V. Bulovic, M. Bawendi, S. Coe-Sullivan, and P. T. Kazlas, "High-efficiency quantum-dot light-emitting devices with enhanced charge injection," Nat. Photonics 7, 407-412 (2013).

2. J. Lim, Y.-S. Park, and V. I. Klimov, "Optical gain in colloidal quantum dots achieved with direct-current electrical pumping," Nat. Mater. 17, 42-49 (2018). 
9:00 - 9:15 Low-loss and Ultra-broadband Silicon Nitride Angled MMI Polarization Splitter (STh1J.5) (https://event.crowdcompass.com/cleo20/activity/4asex98RJp) Ramesh k, Institute for Research in Electronics \& Applied Physics, University of Maryland

9:15 - 9:30 Efficient Second Harmonic Generation in a Si3N4 microring (STh1J.6) (https://event.crowdcompass.com/cleo20/activity/uDItlyUjrH) Xiyuan Lu, National Inst of Standards \& Technology

9:30 - 9:45 Broadband Quasi-Phase-Matching in All-Optically Poled Stoichiometric Silicon Nitride Waveguides (STh1J.7) (https://event.crowdcompass.com/cleo20/activity/w9nsBwPEtt) Edgars Nitiss, Ecole Polytechnique Federale de Lausanne

9:45 - 10:00 A Waveguide-Coupled Colloidal Quantum Dot LED on a Silicon Nitride Platform (STh1J.8) (https://event.crowdcompass.com/cleo20/activity/fwjikoCwXp) Lukas Elsinger, Ghent University - imec
ఓ Paper (https://account.osa.org/eweb/DynamicPage.

ఓ Paper (https://account.osa.org/eweb/DynamicPage.

ఓ Paper (https://account.osa.org/eweb/DynamicPage.

\& Paper (https://account.osa.org/eweb/DynamicPage.

Space-division Multiplexing Transmission (STh1L) (https://event.crowdcompass.com/cleo20/activity/raVNOjUULj) Presider: Giovanni Milione, NEC Laboratories America Inc

Watch Recorded Session (https://osa.zoom.us/rec/share/9fFOK7b76kdlfYXk2kLFXvMnMrbPT6a82nRK-6JfyE1kwZGXo9v8CU_CGVvgd8yH)

(1)

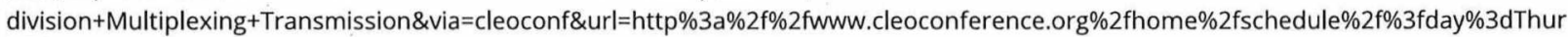
fittps://www.facebook.com/sharer/sharer.php?

u $=$ http $\% 3 a \% 2 f \% 2 f w w w . c l e o c o n f e r e n c e . o r g \% 2 f h o m e \% 2 f s c h e d u l e \% 2 f \% 3 f d a y \% 3 d T h u r s d a y \% 23 S T H 1 L)$ in (http://www.linkedin.com/shareArticle? mini=true\&url=http $\% 3 a \% 2 f \% 2 f w w w . c l e o c o n f e r e n c e . o r g \% 2 f h o m e \% 2 f s c h e d u l e \% 2 f \% 3 f d a y \% 3 d T h u r s d a y \% 23 S T H 1 L \& t i t l e=S p a c e-$

division+Multiplexing+Transmission\&source=CLEO Conference) $\rightarrow$ (http://www.reddit.com/submit? url=http $\% 3 a \% 2$ f $\% 2$ fwww.cleoconference.org $\% 2$ fhome $\% 2$ fschedule $\% 2 f \% 3 f$ day $\% 3$ dThursday $\% 23 \mathrm{STH} 1 \mathrm{~L}$ )

Science \& Innovations

8:00-8:15 Two-channel data transmission on a polarization-maintaining highly elliptical core fiber without MIMO (STh1L.1) (https://event.crowdcompass.com/cleo20/activity/VfmvWtBvTE) Alessandro Corsi, Laval University

8:15 - 8:30 DMD Reduction by Strong Mode Coupling in Few-mode Fibers Using Multi-laser Inscribed LPFGs (STh1L.2) (https://event.crowdcompass.com/cleo20/activity/3gkw4QUExY) Xutao Wang, Key Laboratory of Opto-electronic Information Technical Science of Ministry of Education, School of Precision Instruments and Optoelectronics Engineering, Tianjin University, Tianjin, 300072, China

8:30 - 8:45 Fabry-Perot Filter-Based Mode-Group Demultiplexers (STh1L.3) (https://event.crowdcompass.com/cleo20/activity/tbiXtzORPI) Fatemeh Ghaedi Vanani, University of Central Florida

8:45 - 9:00 Nonlinearity-Aware OAM Mode-Group Multiplexed Transmission over 1-km Ring-Core Fiber with Low High-Order Inter-Mode-Group Crosstalk (STh1L.4) (https://event.crowdcompass.com/cleo20/activity/31I1cKe3IV) Junwei Zhang, Sun Yat-Sen University

9:00 - 9:30 Optimized Multicore Amplifiers for Space-Division Multiplexing Transmission Systems (STh1L.5) (https://event.crowdcompass.com/cleo20/activity/oDKpuGu6sC) Emmanuel Le Taillandier de Gabory, NEC Corporation Invited

9:30 - 9:45 Experimental Demonstration of Record 300-km Orbital Angular Momentum (OAM) Mode-Division Multiplexing Transmission Using a Ring-Core Fiber Recirculating Loop (STh1L.6) (https://event.crowdcompass.com/cleo20/activity/nq6ainxeRf) Hongya Wang, Huazhong University of Science and Techn
ఓ Paper (https://account.osa.org/eweb/DynamicPage

ఓ Paper (https://account.osa.org/eweb/DynamicPage

(1)

\footnotetext{
ఓ Paper (https://account.osa.org/eweb/DynamicPage
}

\$ Paper (https://account.osa.org/eweb/DynamicPage

Paper (https://account.osa.org/eweb/Dynamic Page

ఓ Paper (https://account.osa.org/eweb/DynamicPage

ఓ Paper (https://account.osa.org/eweb/DynamicPage 